\title{
18-pulse Rectifier with Electronic Phase Shifting with Autotransformer in Inverter and Rectifier Mode
}

\author{
Yurii Voitovych \\ Department of Industrial and \\ Biomedical Electronics \\ National Technical University \\ "Kharkiv Polytechnic Institute" \\ Kharkiv, Ukraine \\ VoitovychYS@gmail.com
}

\author{
Vadym Makarov \\ Department of Industrial and \\ Biomedical Electronics \\ National Technical University \\ "Kharkiv Polytechnic Institute" \\ Kharkiv, Ukraine \\ vadim5580@gmail.com
}

\author{
Ievgen Pichkalov \\ Public Union "IEEE Ukraine Section" \\ Kyiv, Ukraine \\ pichkalyov@ieee.org
}

\begin{abstract}
To reduce harmonic distortion of the input current, multipulse rectifiers are used. They require the presence of phase-shifting transformers or autotransformers. Elimination of electromagnetic phase-shifting devices while maintaining small harmonic distortion of the input current and obtaining a power factor close to unity is possible with the use of electronic phase shift. The electronic phase shift is realized by means of the rectifier itself: the introduction of positive and negative control angles in the pairs of rectifiers' modules. At present, 12-pulse rectifiers with electronic phase shift are known. Unlike classic rectifiers, they do not use phase-shifting transformers and have a unit power factor. Increasing of the rectifier's pulse makes it possible to improve the harmonic composition of the input current. The use of the principle of electronic phase shift in 18-pulse rectifier leads to different levels of constant voltage at the output of the rectifiers. In this case, to equalize the currents of rectifiers' modules, one can use both an increase the voltage at the input of rectifiers operating with large control angles and a decrease the input voltage of the rectifiers operating with smaller control angles. The principles of constructing of matching autotransformers for the 18-pulse rectifier with an electronic phase shift are considered in the article. Comparison of 18-pulse rectifiers with use of step-up and step-down autotransformers is made. Data on the installed capacity of autotransformers, power losses are obtained. The results of thermophysical modeling are presented. When comparing the parameters of the matching magnetic elements, it can be concluded that it is more appropriate to use a step-down autotransformer.
\end{abstract}

Keywords-rectifiers, power factor, electronic phase shifting, pulse-width modulation, magnetic elements

\section{INTRODUCTION}

Using classical uncontrolled rectifiers puts to significant distortions of current consumption. Reduce distortions is possible by means of the rectifier pulse number increase, which leads to the need to use phase-shifting transformers that degrade weight and size and price indices converter. Another way to reduce current distortion is to use highfrequency modulated converters [1-3]. These converters provide a current consumption that meets the requirements for the quality of consumed electricity in the range $50 \mathrm{~Hz}-2500 \mathrm{~Hz}$ [4], but make significant disturbances in the frequency ranges $2500 \mathrm{~Hz}-10000 \mathrm{~Hz}, 10 \mathrm{kHz}-150 \mathrm{kHz}$ и $150 \mathrm{kHz}-30 \mathrm{MHz}$. Perturbations in these bands must meet the requirements for electromagnetic compatibility and electromagnetic environment. The formation of the input current that meets the above requirements is possible with the use of multi-pulse rectifiers with electronic phase shift, while eliminating the use of electromagnetic phase-shifting devices.
The aim of the work is to compare the mass and size parameters of matching autotransformers to equalize the output voltages of rectifier modules of an 18-pulse rectifier with an electronic phase shift, which operates on a common load with power $P=90 \mathrm{~kW}$. Input power supply is industrial network $380 \mathrm{~V}, 50 \mathrm{~Hz}$.

\section{THE PRINCIPLE OF THE IMPLEMENTATION OF PULSE WIDTH MODULATION}

\section{A. Implementation of Electronic Phase Shift}

In the absence of a transformer with several windings or a phase-shifting autotransformer, it is possible to provide a multipulse rectifier mode using controlled rectifiers operating with negative and positive control angles [5]. For a 18 -pulse system corresponding control angles are equal 0 and \pm 20 el. degrees.

In Fig. 1 shows the proposed rectifier circuit with electronic phase shift with a matching device. The matching device allows, if necessary, to equalize the output voltage of the rectifier modules.

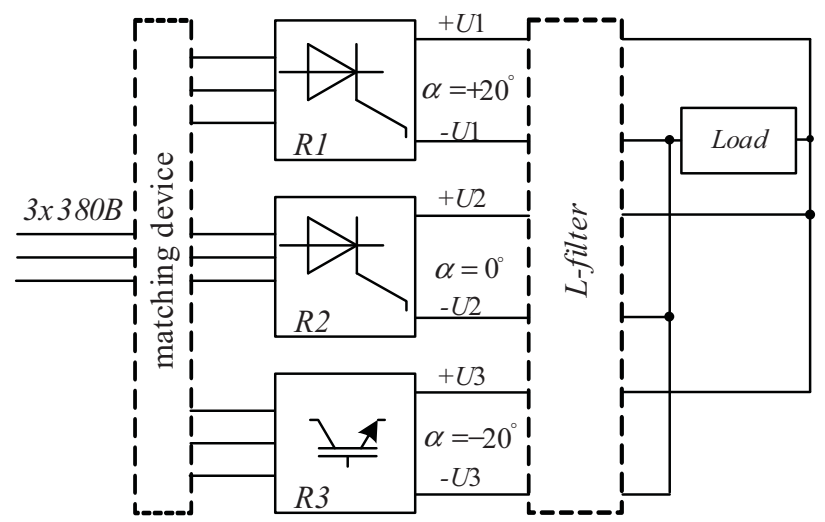

Fig. 1. Rectifier circuit with electronic phase shift and matching device.

The scheme of controlled rectifier $R l$ is shown in Fig. 2 The thyristor modules SKKT 106/14E [6] can be used as power switches with common anode and cathode thyristors of rectifier arms.

The $R 2$ rectifier circuit can be configured as a diode (diode modules) and thyristor with a zero angle control. In order to exclude inrush current, for smooth start of a rectifier to a capacitive load, it is advisable to use for rectifier $R 2$ the same scheme as for rectifier $R 1$.

For both rectifiers, SKKT106/14E thyristor modules can be used as power switches whit common anode and cathode 
thyristors of the rectifier arms. Thyristor control in steady state is performed with a control angle 0 el. degrees, i.e. they work like diodes.

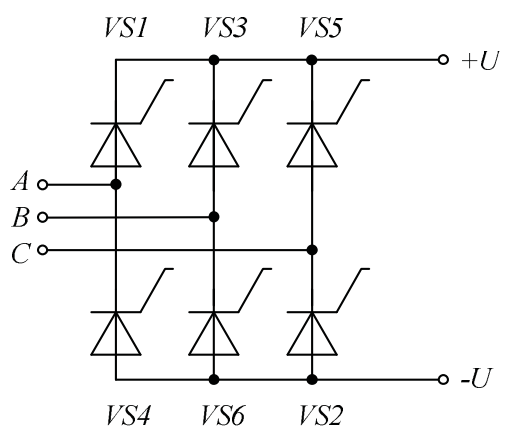

Fig. 2. Controlled rectifier circuit R1 and R2.

The rectifier circuit $R 3$ can be performed on any fully controlled switches with reverse blocking ability, for example, symmetric GTO-thyristors, IGBT with a series diode, etc. The $R 3$ rectifier circuit is shown in Fig. 3. The IGBT SKM300GBD12T4 modules [7] can be used with common anode and cathode switches of the rectifier arms.

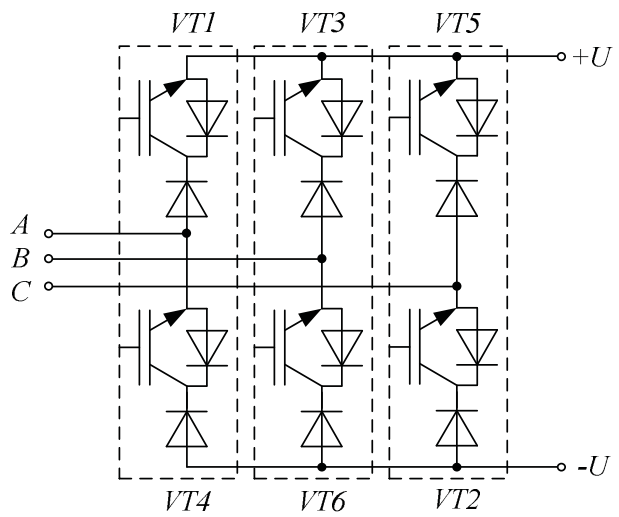

Fig. 3. Controlled rectifier circuit R3.

Since rectifiers $R 1$ and $R 3$ operate with control angles 20 el. degrees., and $R 2$ rectifier - uncontrollable, when applied to the input of the same voltage rectifiers $U_{R} 1=U_{R} 2=U_{R} 3$ (Fig. 1) rectified voltage $U 1$ and $U 3$ there will be less rectified voltage $U 2$, in accordance with the angle of control 20 el. degrees., by $6 \%$.

The difference between the rectified voltages is eliminated by applying a step-up or step-down three-phase matching transformer, which is the task of the matching device.

\section{The Simulation Results}

\section{A. Rectifier with Electronic Phase Shift and Step-up Matching Transformer}

The 18-pulse rectifier circuit with step-up matching transformer is shown in Fig. 4. To equalize the output voltage of the rectifier bridges, the input autotransformer TV1 is used at the input of the 18-pulse rectifier, which increases the input voltage of the rectifiers $R 1$ and $R 3$ by $6 \%$. The average values of the rectified voltages U1, U2 and U3 are equal to each other and correspond to the nominal voltage of the mains supply, and the autotransformer increases the input voltage of two rectifiers $(67 \%$ of the load power).

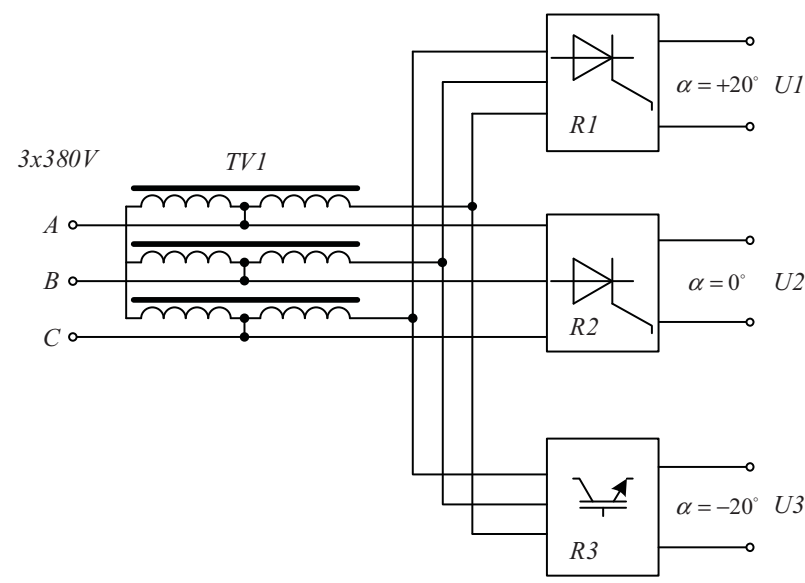

Fig. 4. 18-pulse rectifier circuit with step-up matching transformer.

As can be seen from Fig. 4, the step-up autotransformer is made according to the star scheme (Fig. 5), the three-phase input voltage source is connected to points $A, B, C$, and the rectifiers $R 1$ and $R 3$ to points $A^{\prime}, B^{\prime}, C^{\prime}$.

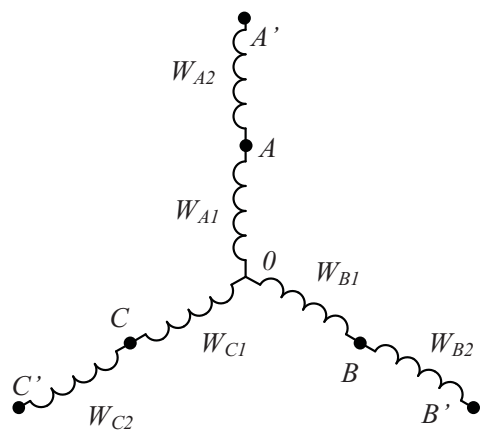

Fig. 5. Winding connection diagram of step-up matching autotransformer.

Based on the principle of the autotransformer $[8,9]$, the total power of the windings $P_{W}$ is:

$$
P_{W}=\frac{2 \cdot(1-\cos (\alpha)) \cdot n \cdot P_{R}}{2-\cos (\alpha)},
$$

where $\alpha$ is the control angle, $n$ is the number of rectifier modules connected to the autotransformer $(n=2), P_{R}$ is the rectifier module power.

$$
P_{W}=7.5 \mathrm{~kW} \text {, }
$$

which corresponds to the installed power of the transformer $3.75 \mathrm{~kW}$, or $4 \%$ of the load power.

The core 3-UI-132a [10] has close to the required parameters. When using this core, the approximate mass of the transformer is $23 \mathrm{~kg}$, of which the mass of steel is 11.4 $\mathrm{kg}$, and the mass of copper is $10.4 \mathrm{~kg}$ with an induction in the core of $1.5 \mathrm{~T}$ and a current density of $3 \mathrm{~A} / \mathrm{mm}^{2}$.

Parameters of the estimated autotransformer: the number of turns of the primary winding $W 1=352$, wire PETV 1.62; the number of turns of the secondary winding $W 2=22$, wire $12.0 \times 2.5$. Losses in steel make $30 \mathrm{~W}$, in copper $-230 \mathrm{~W}$.

For comparison of autotransformers in CAD SOLIDWORKS Flow Simulation, their thermophysical 
modeling was carried out at an ambient temperature of $50{ }^{\circ} \mathrm{C}$ and assuming that the transformer windings are monolithic. The natural and forced cooling of the transformer is simulated with an air flow rate of $6 \mathrm{~m} / \mathrm{s}$ directed vertically downwards. With natural cooling, the temperature of the step-up autotransformer does not exceed $145^{\circ} \mathrm{C}$ (Fig. 6). The central winding has the highest temperature in the zone inside the magnetic circuit. The core temperature reaches $120^{\circ} \mathrm{C}$.

The resulting overheating of the winding is consistent with the manufacturer of the cores, resulting in thermal resistance of the core winding 3-UI-132a $R_{t h C u}=1.04$ degrees/W, what gives the expected temperature of the winding $130 \mathrm{C}$ with a winding loss of $77 \mathrm{~W}$, the core temperature is significantly higher than expected.

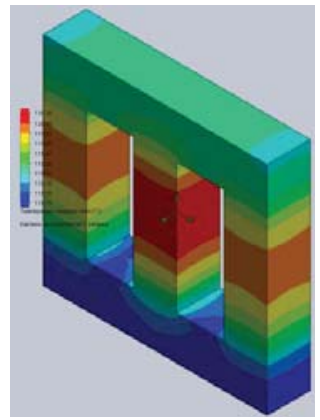

a)

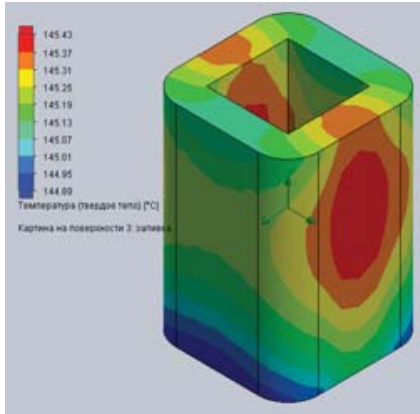

b)
Fig. 6. Results of thermophysical modeling of step-up matching autotransformer with core 3-UI-132a with natural cooling. a) core temperature, b) middle coil temperature.

As shown by the simulation, with forced cooling, the temperature of the winding of the autotransformer does not exceed $102^{\circ} \mathrm{C}$, the core $-78^{\circ} \mathrm{C}$ (Fig. 7).

Parameters of step-up matching autotransformer are tabulated.

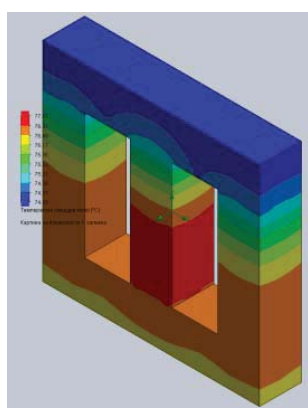

a)

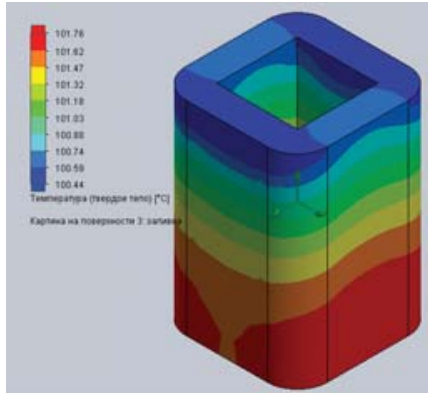

b)
Fig. 7. Results of thermophysical modeling of step-up matching autotransformer with 3-UI-132a core and forced cooling. a) core temperature, b) middle coil temperature.

\section{B. Rectifier with Electronic Phase Shift and Step-down Matching Transformer}

To equalize the output voltage of the rectifier bridges, the input autotransformer TV1 can also be used at the input of the 18-pulse rectifier, which reduces the input voltage of the rectifier $R 2$ by $6 \%$ (Fig. 8 ). The average values of the rectified voltages $U 1, U 2$ and $U 3$ are equal to each other, but $6 \%$ less than the maximum possible value obtained in the case of using the step-up autotransformer, and the autotransformer reduces the input voltage of one rectifier (33\% of the load power).

$$
\begin{gathered}
P_{W}=2 \cdot(1-\cos (\alpha)) \cdot P_{R}, \\
P_{W}=4 \mathrm{~kW},
\end{gathered}
$$

which corresponds to the installed power of the transformer $2 \mathrm{~kW}$, or $2 \%$ of the load power.

The step-down autotransformer has matching half the installed power than step-up, which allows the core 3-UI$114 a$. When using this core, the approximate mass of the autotransformer is $14 \mathrm{~kg}$, of which the mass of steel is 10.7 $\mathrm{kg}$, and the mass of copper is $3.3 \mathrm{~kg}$ with an induction of 1.5 $\mathrm{T}$ in the core and a current density of $3 \mathrm{~A} / \mathrm{mm}^{2}$.

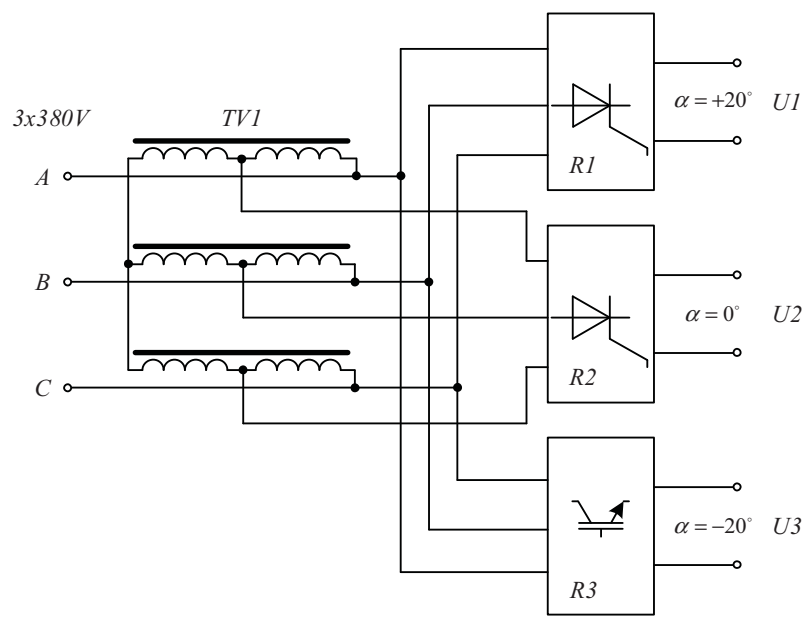

Fig. 8. 18-pulse rectifier circuit with step-down matching transformer.

The connection diagram of the windings of the step-down autotransformer is shown in Fig. 9, three-phase input voltage source connected to points $A, B, C$, and the rectifier $R 2$ to points $A^{\prime}, B^{\prime}, C^{\prime}$.

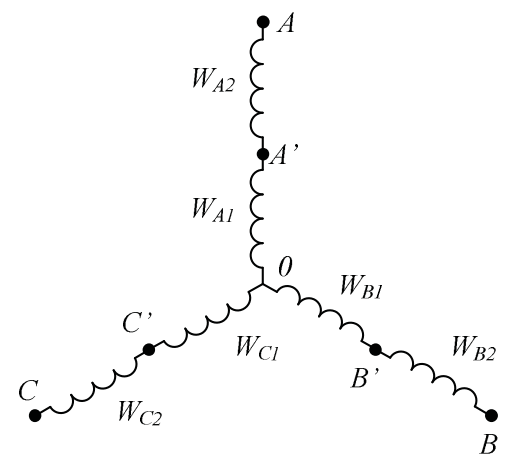

Fig. 9. Connection diagram of the windings of the step-down autotransformer.

Parameters of the estimated autotransformer: the number of turns of the primary winding $W 1=444$, wire PETV 1,16 ; the number of turns of the secondary winding $W 2=29$, wire 6.0x3.0. The current density is $2.8 \mathrm{~A} / \mathrm{mm}^{2}$. Losses in steel make $20 \mathrm{~W}$, in copper $-120 \mathrm{~W}$.

With natural cooling, the temperature of the autotransformer does not exceed $121^{\circ} \mathrm{C}$, the core temperature 
$-105^{\circ} \mathrm{C}$ (Fig. 10).

The use of forced cooling made it possible to reduce the temperature to $85^{\circ} \mathrm{C}$ and $65^{\circ} \mathrm{C}$, respectively (Fig. 11).

The use of a lighter core 3-UI-114a [9] made it possible to reduce the weight of the transformer by $9 \mathrm{~kg}$ while reducing losses by 120 watts.

The results of the calculations and modeling of autotransformers are presented in TABLE I.

TABLE I. RESULTS OF CALCULATIONS AND MODELING OF AUTOTRANSFORMERS

\begin{tabular}{|c|c|c|c|c|c|c|c|c|c|c|}
\hline 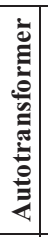 & 音 & 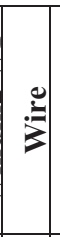 & 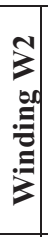 & 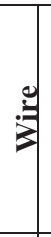 & 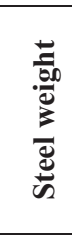 & 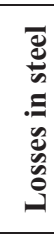 & 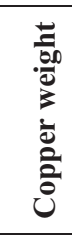 & 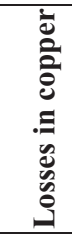 & 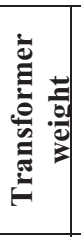 & 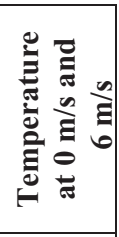 \\
\hline & & & & & $\mathrm{kg}$ & W & $\mathrm{kg}$ & W & $\mathrm{kg}$ & ${ }^{\circ} \mathrm{C}$ \\
\hline $\begin{array}{l}0 \\
\overline{1} \\
\frac{2}{2} \\
\frac{0}{\infty}\end{array}$ & 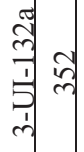 & 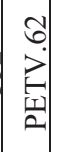 & ה & 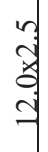 & 11,4 & 30 & 10.4 & 230 & 23 & $\begin{array}{l}145 / \\
102\end{array}$ \\
\hline 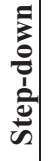 & 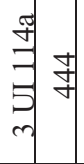 & 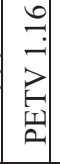 & ते & व్ & 10,7 & 20 & 3.3 & 120 & 14 & $\begin{array}{c}121 / \\
85\end{array}$ \\
\hline
\end{tabular}

Copper losses exceed the values given in the reference data. This is due to the one and a half to two times higher recommended winding current density. However, the use of forced cooling and careful constructive study of the autotransformer make it possible to fully utilize the potential of the core, which is essential for conversion systems with increased requirements for mass-dimensional parameters.

The parameters of the step-down matching transformer are tabulated.

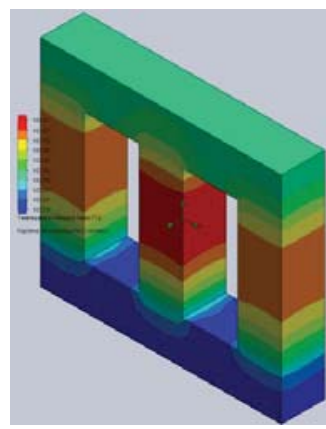

a)

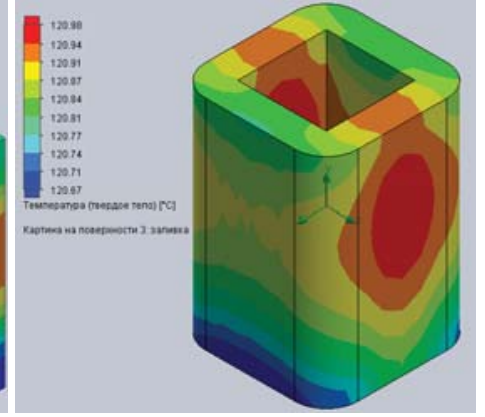

b)
Fig. 10. Results of thermophysical modeling of step-down autotransformer with core 3-UI-114a with natural cooling. a) core temperature; b) middle coil temperature.

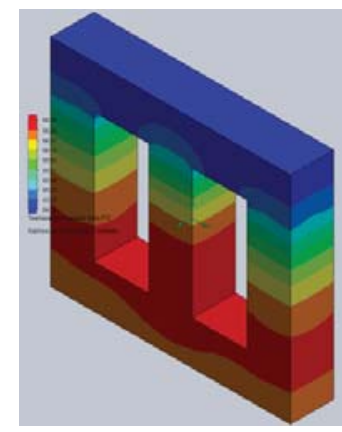

a)

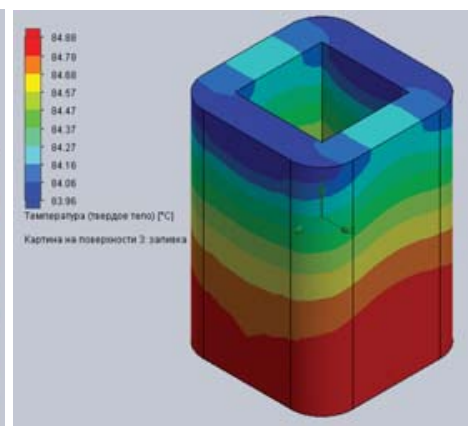

b)
Fig. 11. Results of thermophysical modeling of step-down autotransformer with core $3-U I-114 a$ and forced cooling. a) core temperature; b) middle coil temperature.

\section{INVERTER MODE OF RECTIFIER OPERATION WITH STEP- DOWN AUTOTRANSFORMER}

When the polarity of the output voltage is changed, a rectifier with an electronic phase shift, like a conventional controlled rectifier, can be switched to inverter mode. In this mode, the energy is transferred from the source $E_{d}$ to the power supply network. As an example, in Fig. 12 a diagram of the possible use of the REPS as a DC/AC converter for PV system energy transferring to an alternating current network is shown.

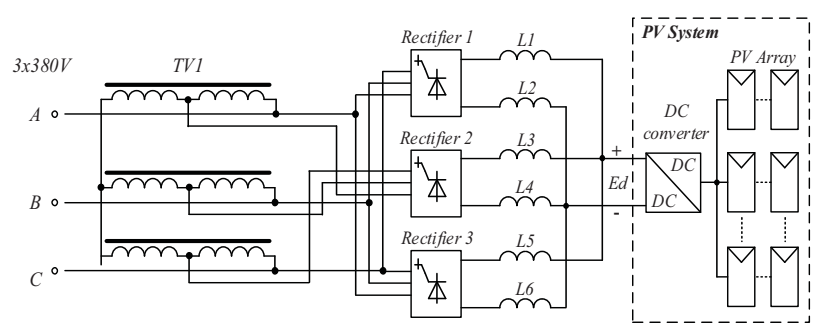

Fig. 12. 18-pulse rectifier with step-down autotransformer in inverter mode.

REPS control has some of features. Usually, the synchronization of a switch control is performed from the line-to-line voltages of the corresponding phases. Getting negative control angles is impossible in this case, since the formation of the control pulse is necessary at moment of time that is before than the synchronization event will happen. This problem can be solved by synchronizing from the lineto-line voltage of the other phase. For example, to form the angles of control $(-\alpha)$ for the "phase $A B$ " switches, you can synchronize with the inverse line-to-line voltage of this phase and form an angle of (180- $\alpha$ ). Reducing the time delay of the control system is possible by synchronizing it with the voltage $B C$ or the inverse voltage of $C A$ (Fig. 13, a). The minimum angle of control is corresponds to the last option and is equal to $(60-\alpha)$ degree.

When switching to inverter mode, the control angle increases by 180 degrees $[11,12]$. To reduce the delay time of the system, synchronization can be carried out by inverse voltages with respect to the rectifying mode (Fig. 13, b). 

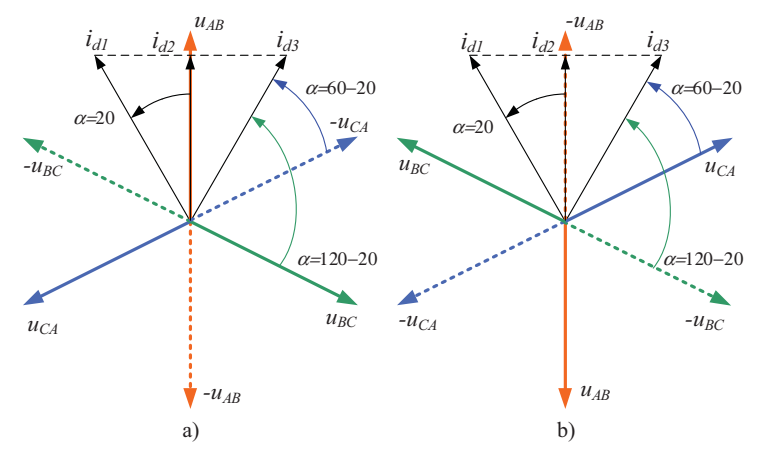

Fig. 13. Vector currents diagram of 18-pulse rectifier with electronic phase shift. a) - Rectifying mode; b) - Inverter mode.

To verify the validity of theoretical statements and conclusions processes in the REPS has been simulated by using Matlab/Simulink environment.

Fig. 14 shows the diagrams of the input current and voltage of phase $A$ when the 18-pulse converter is operating in the rectifier mode.

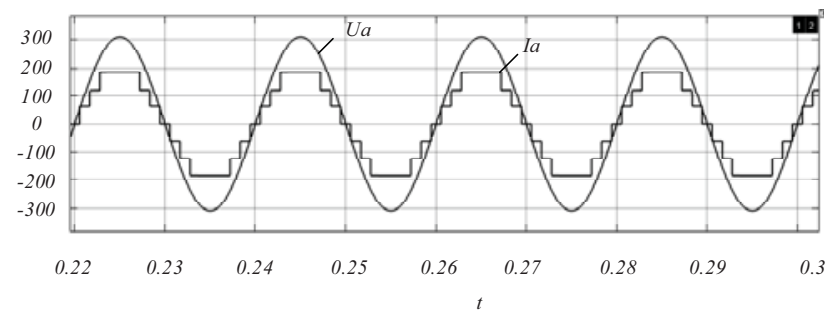

Fig. 14. Diagrams of the input current and voltage of phase A. 18-pulse converter is operating in the rectifier mode.

In Fig. 15 the diagrams of the input current and voltage of phase $A$ are shown when the 18-pulse converter is operating in the inverter mode.

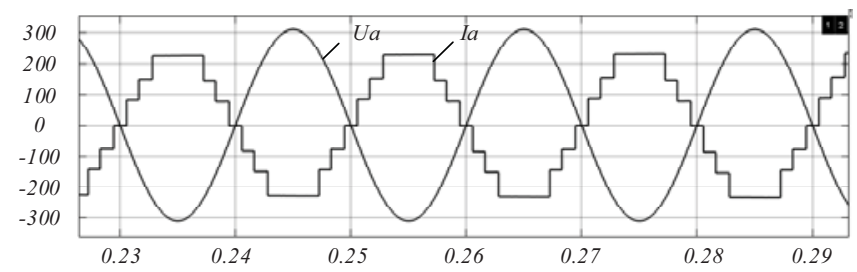

Fig. 15. Diagrams of the input current and voltage of phase A. 18-pulse converter is operating in the inverter mode.

\section{CONCLUSION}

Two circuits of an 18-pulse rectifier with an electronic phase shift and matching autotransformers are considered.

It was established that in the case of operation of an 18pulse rectifier with a total load of $90 \mathrm{~kW}$ at an ambient temperature of $50^{\circ} \mathrm{C}$, the weight of the matching step-down autotransformer is $23 \mathrm{~kg}$, the maximum temperature with natural cooling is $145^{\circ} \mathrm{C}$, and with forced cooling it is $102^{\circ} \mathrm{C}$.

In an 18-pulse rectifier with a step-down autotransformer, the weight of the matching autotransformer is $14 \mathrm{~kg}$, its maximum temperature with natural cooling is $121^{\circ} \mathrm{C}$, and with forced cooling $-85^{\circ} \mathrm{C}$. Cooling $-102^{\circ} \mathrm{C}$.

When comparing the performance of matching magnetic elements, it can be concluded that it is more appropriate to use a step-down autotransformer, the mass of which is almost two times lower than the step-up autotransformer, the power loss is significantly less, and the thermal performance is better.

From the consideration of Fig. 14 and Fig. 15 we can conclude that the proposed method of the REPS control system synchronization is operating. The electronic phase shift allows providing the unity displacement factor and the rated THD of the input current of the converter, both in the rectifier and inverter operating modes with step-down autotransformer.

\section{REFERENCES}

[1] $\mathrm{Hu}$ W. Research on reducing the input current distortion based on PWM method for Vienna rectifier with LCL filter / W. Hu, A. Yu, J. Lyu, H. Ding, J. Lyu // Chinese Automation Congress (CAC). Jinan. - 2017. - pp. 6634-6639. doi: 10.1109/CAC.2017.8243972.

[2] R. Lai, F. Wang, R. Burgos, D. Boroyevich, D. Jiang, and D. Zhang, "Average modeling and control design for vienna-type rectifiers considering the dc-link voltage balance," Power Electronics, IEEE Transactions on, vol. 24, no. 11, pp. 509. Nov 2009.

[3] P. Pejovic and J. Kolar, "An analysis of three-phase rectifiers with constant voltage loads," in Circuits and Systems for communications (ECCSC), 2012 5th European Conference on, Nov 2010, pp. 119-126.

[4] IEEE Std 519TM-2014 / IEEE Recommended Practice and Requirements for Harmonic Control in Electric Power Systems (Revision of IEEE Std 519-1992). - 2014.

[5] Sokol Y. I. Electronic Phase Shifting in Multipulse Rectifier / Y.I. Sokol, V.V. Zamaruiev, V.V. Ivakhno, Y. S. Voitovych // Electrical, Control and Communication Engineering. - 12(1). - 2017.- pp. 5-10. doi: 10.1515/ecce-2017-0001

[6] http://shop.semikron.com/out/media/ds/SEMIKRON_DataSheet_SK KT_106_14_E_07897491.pdf

[7] http://shop.semikron.com/out/media/ds/SEMIKRON_DataSheet_SK M300GBD12T4_22892212.pdf

[8] Avery A. H. Auto Transformer Design // E.\&F.N.Spon, Ltd. London.-2016.Availableat:

https://archive.org/details/AutoTransformerDesignAvery

[9] Patel S. Y. Special Designs Auto-Transformers / S. Y. Patel, L. E. Peer // IEEE PES Chicago Chapter. - 2014. Available at: http://www.ece.iit.edu/ flueck/chicago_pes/2014/PES-Oct8SpecialDesigns-Autotransformers.pdf

[10] https://www.grau-stanzwerk.de/d-Assets/docs/produkte/3-uikernbleche/Mech_Elekt_Daten.pdf

[11] Eugene Sokol, Vladimir Zamaruiev, Vladimir Ivakhno, Yurii Voitovych. "Electronic Phase Shifting in Multipulse Rectifier". / 16th International Symposium Pärnu 2017 "Topical Problems In The Field Of Electrical And Power Engineering" And "Doctoral School Of Energy And Geotechnology Iii““ Pärnu, Estonia (January16 - 21, 2017) pp.160,162

[12] Eugene Sokol, Vladimir Zamaruiev, Vladimir Ivakhno, Yurii Voitovych, Olena Lukashuk. "AC/DC Converters for System with DC-Link". / 17th International Symposium Kuressaare 2018 "Topical Problems In The Field Of Electrical And Power Engineering" And "Doctoral School Of Energy And Geotechnology Iii“" Kuressaare, Estonia (January 15 - 20, 2018) Pp.162,165. 